\title{
Two-component formulation of the Wheeler-DeWitt equation
}

Ali Mostafazadeh

Citation: Journal of Mathematical Physics 39, 4499 (1998); doi: 10.1063/1.532522

View online: http://dx.doi.org/10.1063/1.532522

View Table of Contents: http://aip.scitation.org/toc/jmp/39/9

Published by the American Institute of Physics

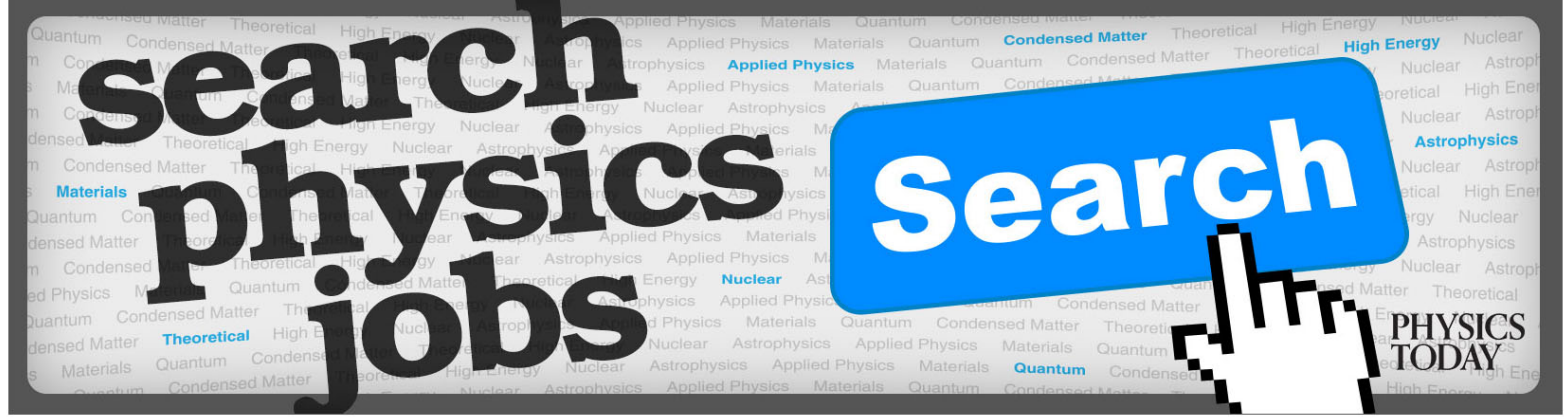




\title{
Two-component formulation of the Wheeler-DeWitt equation
}

\author{
Ali Mostafazadeh ${ }^{\text {a) }}$ \\ Theoretical Physics Institute, University of Alberta, Edmonton, Alberta T6G 2J1, Canada \\ and Department of Mathematics, Koç University, Istinye 80860, Istanbul, Turkey
}

(Received 30 January 1997; accepted for publication 27 April 1998)

The Wheeler-DeWitt equation for the minimally coupled Friedman-RobertsonWalker-massive-scalar-field minisuperspace is written as a two-component Schrödinger equation with an explicitly "time"-dependent Hamiltonian. This reduces the solution of the Wheeler-DeWitt equation to the eigenvalue problem for a nonrelativistic one-dimensional harmonic oscillator and an infinite series of trivial algebraic equations whose iterative solution is easily found. The solution of these equations yields a mode expansion of the solution of the original Wheeler-DeWitt equation. Further analysis of the mode expansion shows that in general the solutions of the Wheeler-DeWitt equation for this model are doubly graded, i.e., every solution is a superposition of two definite-parity solutions. Moreover, it is shown that the mode expansion of both even- and odd-parity solutions is always infinite. It may be terminated artificially to construct approximate solutions. This is demonstrated by working out an explicit example which turns out to satisfy DeWitt's boundary condition at initial singularity. (c) 1998 American Institute of Physics. [S0022-2488(98)01209-2]

\section{INTRODUCTION}

It is well-known that the Wheeler-DeWitt equation for the minimally coupled FriedmanRobertson-Walker (FRW)-massive-scalar-field (the scalar field is assumed to be homogeneous) minisuperspace can be written in the form of the Klein-Gordon equation (this equation corresponds to simplest choice of factor ordering) in $(1+1)$ dimensions, ${ }^{1}$

$$
\left[-\frac{\partial^{2}}{\partial \alpha^{2}}+\frac{\partial^{2}}{\partial \phi^{2}}+\kappa e^{4 \alpha}-m^{2} e^{6 \alpha} \phi^{2}\right] \psi(\alpha, \phi)=0,
$$

where $\alpha=\ln a, a$ is the scale factor of the FRW metric

$$
\begin{gathered}
d s^{2}=-d t^{2}+a^{2}(t)\left[d r^{2}+S_{\kappa}(r)\left(d \theta^{2}+\sin ^{2} \theta d \varphi^{2}\right)\right], \\
S_{\kappa}:=\left\{\begin{array}{l}
\sinh r \text { for } \kappa=-1: \quad \text { open universe } \\
r \quad \text { for } \kappa=0: \text { flat universe } \\
\sin r \text { for } \kappa=1: \quad \text { closed universe, }
\end{array}\right.
\end{gathered}
$$

and $\phi$ is a real scalar field of mass $m$. It is also well-known that unlike the massless case ${ }^{2}$ exact solutions of this equation are not known. The approximate solutions are usually obtained using the semiclassical Wentzel-Kramers-Brillouin (WKB) methods. ${ }^{3}$

Recently, I have used a two-component formulation of the Klein-Gordon equation to study the geometrical phases induced on a Klein-Gordon field due to a dynamical background spacetime geometry and electromagnetic or scalar interactions. ${ }^{4}$ In the present article, I shall try to apply the same approach for the treatment of the Wheeler-DeWitt equation (1).

${ }^{a}$ Electronic mail: amostafazadeh@ku.edu.tr 


\section{TWO-COMPONENT FORM OF THE WHEELER-DEWITT EQUATION}

The idea of the two-component formalism ${ }^{5}$ is to transform the Klein-Gordon equation which is of second order in time-derivative, into a system of two coupled differential equations which are first order in the time-derivative. The latter may be viewed as a Schrödinger equation. In this way one can apply the well-developed techniques of solving the Schrödinger equation to obtain solutions of the Klein-Gordon equation. The application of the two-component formalism for the Klein-Gordon equation is described in detail in Ref. 4. Here I only include the results which are relevant for the solution of Eq. (1).

Equation (1) can be expressed in the form:

$$
\left[\eta^{\mu \nu} \frac{\partial}{\partial X^{\mu}} \frac{\partial}{\partial X^{\nu}}+V(X)\right] \psi=0
$$

where $\mu, \nu=0,1,\left(\eta^{\mu \nu}\right):=\operatorname{diag}(-1,1), X^{0}:=\alpha, X^{1}:=\phi$, and

$$
V(X):=\kappa e^{4 X^{0}}-m^{2} e^{6 X^{0}}\left(X^{1}\right)^{2}=\kappa e^{4 \alpha}-m^{2} e^{6 \alpha} \phi^{2}=\kappa a^{4}-m^{2} a^{6} \phi^{2} .
$$

Hence, $\alpha$ plays the role of "time.' Furthermore, defining

$$
\Psi:=\frac{1}{\sqrt{2}}\left(\begin{array}{c}
\psi+i \dot{\psi} \\
\psi-i \psi
\end{array}\right)
$$

one can easily check that Eq. (2) is equivalent to the Schrödinger equation

$$
i \dot{\Psi}=H \Psi
$$

with the explicitly time-dependent Hamiltonian:

$$
H=\frac{1}{2}\left(\begin{array}{cc}
1+D & -1+D \\
1-D & -1-D
\end{array}\right)
$$

where a dot represents differentiation with respect to $X^{0}=\alpha$ and

$$
D:=-\frac{\partial^{2}}{\partial\left(X^{1}\right)^{2}}-V=-\frac{\partial^{2}}{\partial \phi^{2}}-V .
$$

The two-component Hamiltonian (6) has the following remarkable properties.

(1) It belongs to the (dynamical) Lie algebra $\operatorname{su}_{s}(1,1) \otimes \mathrm{su}_{u}(1,1)$. Here $\mathrm{su}_{s}(1,1)$ is the standard representation of $\operatorname{su}(1,1)$ with the basis

$$
K_{3}=J_{3}=\frac{1}{2} \sigma_{3}, \quad K_{a}=i J_{\alpha}=\frac{i}{2} \sigma_{a} \quad(a=1,2),
$$

where $\sigma_{i}$ are Pauli matrices, and $\mathrm{su}_{u}(1,1)$ denotes the unitary representation of $\mathrm{su}(1,1)$ constructed out of the position $x$ and momentum $p$ operators of the one-dimensional quantum mechanics, with the basis $\left\{\left(x^{2}-p^{2}\right) / 4,\left(x^{2}+p^{2}\right) / 4,(x p+p x) / 4\right\} .{ }^{6}$ This is easily seen, by writing $H$ in the form $H=K_{-}+K_{+} D$, where $K_{ \pm}:=K_{3} \pm K_{2}$, and observing that the operator $D$ belongs to $\operatorname{su}_{u}(1,1)$.

(2) It is the square root of the operator $D$, in the sense that it satisfies

$$
H^{2}=\left(\begin{array}{cc}
D & 0 \\
0 & D
\end{array}\right)=D
$$

This is reminiscent of Dirac's treatment of the noninteracting Klein-Gordon equation in $(3+1)$-dimensional Minkowski space, where the Dirac operator plays the role of the square root of the Klein-Gordon operator.

Next let us examine the eigenvalues $E_{n}$ and eigenvectors $\Psi_{n}$ of the Hamiltonian $H$. In order to have a well-posed eigenvalue problem for $H$, one should discuss the properties of the space of 
two-component wave functions $\mathscr{H}$. This will be done in Sec. III. Here we suffice to note that as a vector space $\mathscr{H}$ is the inner sum of two vector subspaces each of which is isomorphic (as Hilbert spaces) to $L^{2}(\mathbb{R})$. In view of this observation and the particular form of the Hamiltonian $H$, the formal solution of the eigenvalue equation

$$
H \Psi_{n}=E_{n} \Psi_{n},
$$

for $H$ is given by

$$
\Psi_{n}=\frac{1}{\sqrt{2}}\left(\begin{array}{c}
1+E_{n} \\
1-E_{n}
\end{array}\right) \Phi_{n}, \quad D \Phi_{n}=E_{n}^{2} \Phi_{n}
$$

This shows that the eigenvalue equation (9) for $H: \mathscr{H} \rightarrow \mathscr{H}$ is in fact equivalent to the eigenvalue equation (10) for $D: L^{2}(\mathbb{R}) \rightarrow L^{2}(\mathbb{R})$. Since the latter is a well-posed eigenvalue equation in the Hilbert space $L^{2}(\mathbb{R})$, so is the former. In particular, the eigenvectors $\Psi_{n}$ do belong to $\mathscr{H}$.

Next let use substitute Eq. (7) in the second equation in (10). This yields

$$
\left[-\frac{1}{2} \frac{\partial^{2}}{\partial \phi^{2}}+\frac{1}{2} \omega^{2} \phi^{2}\right] \Phi_{n}=\mathscr{C}_{n} \Phi_{n}
$$

where $\omega:=m e^{3 \alpha}=m a^{3}$ and $\mathscr{C}_{n}:=\left(E_{n}^{2}+\kappa e^{4 \alpha}\right) / 2=\left(E_{n}^{2}+\kappa a^{4}\right) / 2$. Equation (11) is the eigenvalue equation for a simple harmonic oscillator with frequency $\omega$ and unit mass. Hence:

$$
\begin{gathered}
\mathscr{E}_{n}=\omega\left(n+\frac{1}{2}\right)=m a^{3}\left(n+\frac{1}{2}\right), \\
\Phi_{n}=N_{n} H_{n}(\sqrt{\omega} \phi) e^{-\frac{1}{2} \omega \phi^{2}}=N_{n} H_{m}\left(\sqrt{m a^{3}} \phi\right) e^{-\frac{1}{2} m a^{3} \phi^{2}},
\end{gathered}
$$

where $H_{n}$ are Hermite polynomials and $N_{n}$ are normalization constants. The latter are determined by the requirement that $\Phi_{n}$ be normalized with the usual $L^{2}(\mathbb{R})$ norm. This leads to

$$
N_{n}=\left[\frac{\omega}{\pi\left(2^{n} n !\right)^{2}}\right]^{1 / 4}=\left[\frac{m a^{3}}{\pi\left(2^{n} n !\right)^{2}}\right]^{1 / 4} .
$$

Note that $\Phi_{n}$ and consequently $\Psi_{n}$ tend to zero as $a \rightarrow 0$ or $a \rightarrow \infty$.

Substituting Eq. (12) in the expression for $\mathscr{E}_{n}$, one has

$$
E_{ \pm n}= \pm \sqrt{\omega(2 n+1)-\kappa e^{4 \alpha}}= \pm \sqrt{m e^{3 \alpha}(2 n+1)-\kappa e^{4 \alpha}}= \pm a \sqrt{m a(2 n+1)-\kappa a^{2}} .
$$

Thus, for $\kappa=-1$ or $0, E_{ \pm n}$ are real, whereas for $\kappa=1$ and $a>m$, there are eigenvectors $\Psi_{ \pm n}$ with imaginary eigenvalue. They correspond to the integers $n$ satisfying $m(2 n+1)<a$.

So far, I have given the solution for the eigenvalue problem for the Hamiltonian $H$. The eigenvectors of $H$, however, do not solve the Schrödinger equation. This is mainly because $H$ is explicitly time dependent. The general solution of the Schrödinger equation for an arbitrary explicitly time-dependent Hamiltonian is not known. However, in the cases where the eigenvalue problem for the Hamiltonian is solvable, there are techniques based on the adiabatic approximation $^{7-9}$ which may be used to obtain approximate and occasionally exact solutions. These techniques can be employed if the Hamiltonian is self-adjoint. In order to examine the self-adjointness of the Hamiltonian $H$, one first needs a notion of an inner product on the space $\mathscr{H}$ of two-component wave functions $\Psi$.

\section{HILBERT SPACE STRUCTURE OF $\mathscr{H}$}

As a vector space, the space $\mathscr{H}$ of two-component wave functions is isomorphic to $L^{2}(\mathbb{R})$ $\oplus L^{2}(\mathbb{R})$. Here by the vector space $L^{2}(\mathbb{R}) \oplus L^{2}(\mathbb{R})$, I mean the vector space inner sum of two copies of $L^{2}(\mathbb{R})$, not the Hilbert space inner sum of these spaces where the sum is also endowed with a canonical inner product. Furthermore, in what follows I allow for negative norms and the terms "inner product" and "Hilbert space" refer to a Hermitian bilinear (sesquilinear) form and 
a vector space endowed with such a form, respectively. [A Hermitian bilinear or sesquilinear form on a complex vector space $V$ is a function $\mu: V \times V \rightarrow \mathrm{C}$ which is linear in its second variable and satisfies $\mu\left(v_{1}, v_{2}\right)=\mu\left(v_{2}, v_{1}\right)^{*}$ where* denotes complex conjugation.]

The relation $\mathscr{H}=L^{2}(\mathbb{R}) \oplus L^{2}(\mathbb{R})$ may be viewed as a restriction on the solutions of the Wheeler-DeWitt equation, since for finite $\alpha$ the elements of $L^{2}(\mathbb{R}) \oplus L^{2}(\mathbb{R})$ vanish at "spatial" infinity, i.e., $\phi= \pm \infty$. This choice seems, however, to be a natural and at the same time a rather general one. It is only restrictive as far as the boundary condition at spatial infinity is concerned. It is still quite general, for it does not correspond to any fixed boundary conditions at $\alpha= \pm \infty$. Furthermore, although $\mathscr{H}$ has two vector subspaces with $L^{2}$ inner products, an inner product on $\mathscr{H}$ is not a priori fixed.

An inner product (sesquilinear form) on $\mathscr{H}$ may be given by the inner product on $L^{2}(\mathbb{R})$ and a Hermitian two-by-two matrix $h$. A particularly advantageous choice for $h$ is $h=\sigma^{3}=\operatorname{diag}(1$, $-1)$, i.e.,

$$
(\Psi, \widetilde{\Psi}):=\left\langle\Psi^{1} \mid \widetilde{\Psi}^{1}\right\rangle-\left\langle\Psi^{2} \mid \widetilde{\Psi}^{2}\right\rangle,
$$

where $\langle\mid\rangle$ denotes the inner product on $L^{2}(\mathbb{R})$. This choice, on the one hand, renders $H$ " selfadjoint" in the sense that for all $\Psi, \widetilde{\Psi} \in \mathscr{H}$,

$$
(\Psi, H \widetilde{\Psi})=(H \Psi, \widetilde{\Psi}) .
$$

[Note that the adjoint of a linear operator is well-defined if one has an inner product which defines a positive definite norm. This is because in the definition of the adjoint of a linear operator one uses the Riesz lemma whose proof makes use of the positive definiteness of the norm. ${ }^{10}$ Here the term "self-adjoint" refers to the validity of Eq. (16).] On the other hand, it reduces to the Klein-Gordon inner product (for more details see Ref. 4) for the solutions (4) of the Schrödinger equation (5).

Adopting this inner product on $\mathscr{H}$, one can compute

$$
\left(\Psi_{ \pm m}, \Psi_{ \pm n}\right)=\left(E_{ \pm m}^{*}+E_{ \pm n}\right) \delta_{m n} .
$$

Here I have used (10) and the fact that $\Phi_{n}$ are orthonormal. In particular, $\left(\Psi_{ \pm n}, \Psi_{ \pm n}\right)$ $=2 \operatorname{Re}\left(E_{ \pm n}\right)= \pm 2\left|\operatorname{Re}\left(E_{n}\right)\right|$. Hence, the eigenvectors $\Psi_{ \pm n}$ with imaginary eigenvalues (if they exist) have zero norm; they are null vectors. This is of course to be expected since the Hamiltonian satisfies Eq. (16). (Note that it is the nonpositive definiteness of the corresponding norm that allows for imaginary eigenvalues and null eigenvectors to exist.) Furthermore, note that the null eigenvectors are only present for the $\kappa=1$ case.

Another interesting consequence of Eq. (17) is that

$$
\left(\Psi_{ \pm n}, \Psi_{\mp n}\right)=\left\{\begin{array}{l}
0 \quad \text { for } E_{n} \text { real } \\
E_{\mp n} \text { for } E_{n} \text { imaginary. }
\end{array}\right.
$$

Hence, if we view $\mathscr{H}$ as the linear span of $\Psi_{ \pm n}$, it can be orthogonally decomposed as

$$
\mathscr{H}=\mathscr{H}^{(-)} \oplus \mathscr{H}^{(0)} \oplus \mathscr{H}^{(+)},
$$

where $\mathscr{H}^{(-)}, \mathscr{H}^{(0)}$, and $\mathscr{H}^{(+)}$are the linear span of the negative-norm, null, and positive-norm eigenvectors. Note that this decomposition is time dependent (it depends on $a=e^{a}$ ), and not every null vector belongs to $\mathscr{H}^{(0)}$. Furthermore, Eq. (18) implies that the basis $\left\{\Psi_{n}\right\}$ (respectively, $\left\{\Psi_{-n}\right\}$ ) of $\mathscr{H}^{(+)}$(respectively, $\mathscr{H}^{(-)}$) is orthogonal, whereas $\mathscr{H}_{0}$ can be orthogonally decomposed into the sum of two-dimensional subspaces $\mathscr{H}_{n}^{(0)}=\operatorname{Span}\left(\Psi_{n} \oplus \Psi_{-n}\right)$ with $n \leqslant(a / m$ $-1) / 2$, namely

$$
\mathscr{H}^{(0)}=\bigoplus_{n=1}^{N} \mathscr{H}_{n}^{(0)}
$$

where $N:=[(a / m-1) / 2]$ is the largest integer part of $(a / m-1) / 2$. 
Although the inner product (15) has a number of interesting properties, in the remainder of this article I shall not assume that $\mathscr{H}$ is endowed with this inner product. In fact, the results presented in the following sections are independent of the choice of the $2 \times 2$ matrix $h$ which determines the inner product on $\mathscr{H}$. I shall only use the well-known Hilbert space properties of the space $L^{2}(R)$.

\section{ADIABATIC APPROXIMATION}

Consider the ansatz

$$
\Psi=e^{i \zeta / N} \Psi_{\mathscr{N}},
$$

for the solution of the Schrödinger equation (5), where $\zeta_{\mathscr{N}}$ is a complex-valued function of $\alpha$, and $\mathscr{N}= \pm n \in \mathbb{Z}$. Plugging (21) in (5) and making use of the eigenvalue equation (9) and the form (10) of the eigenvectors $\Psi_{\mathscr{N}}$, one has:

$$
\begin{aligned}
& {\left[-\left(\dot{\zeta}_{\mathscr{N}}+E_{\mathscr{N}}\right)\left(1+E_{\mathscr{N}}\right)+i \dot{E}_{\mathscr{N}}\right] \Phi_{n}+i\left(1+E_{\mathscr{N}}\right) \dot{\Phi}_{n}=0,} \\
& {\left[-\left(\dot{\zeta}_{\mathscr{N}}+E_{\mathscr{N}}\right)\left(1-E_{\mathscr{N}}\right)-i \dot{E}_{\mathscr{N}}\right] \Phi_{n}+i\left(1-E_{\mathscr{N}}\right) \dot{\Phi}_{n}=0 .}
\end{aligned}
$$

Adding and subtracting both sides of these equations, one finds:

$$
\left(\dot{\zeta}_{\mathscr{N}}+E_{\mathscr{N}}\right) \Phi_{n}-i \dot{\Phi}_{n}=0, \quad \dot{E}_{\mathscr{N}}=0 .
$$

Here the first equation implies that for $\tilde{n} \neq n, A_{\tilde{n} n}:=i\left\langle\Phi_{\tilde{n}} \mid \Phi_{n}\right\rangle=0$. This requirement together with the second relation in (22) signify the conditions under which the adiabatic approximation would yield an exact solution of the Schrödinger equation. Clearly, $\dot{E}_{\mathscr{N}} \neq 0$ for the case under consideration. Thus the adiabatic approximation cannot be exact. However, approximate solutions may be sought if $\dot{E}_{\mathscr{K}}$ and $A_{\tilde{n} n}$ are small.

Performing the necessary calculations, one can show that for arbitrary non-negative integers $\tilde{n}$ and $n$ :

$$
\begin{gathered}
A_{\tilde{n} n}:=i\left\langle\Phi_{\tilde{n}} \mid \dot{\Phi}_{n}\right\rangle=\frac{3 i}{4}\left[\sqrt{n(n-1)} \delta_{\tilde{n}, n-2}-\sqrt{(\tilde{n})(\tilde{n}-1)} \delta_{\tilde{n}, n+2}\right], \\
\dot{E}_{\mathscr{N}}= \pm \frac{3\left[n+\frac{1}{2}-\frac{2 \kappa a}{3 m}\right] \sqrt{m} a^{3 / 2}}{\sqrt{2 n+1-\frac{\kappa a}{m}}} \text { where } \mathscr{N}= \pm n .
\end{gathered}
$$

Equation (23) indicates that the adiabatic approximation is not valid, i.e., the solutions of the form (21) do not exist.

Another consequence of Eq. (23) is that in a mode expansion of a solution $\Psi$ of Eq. (5), the even modes $\Psi_{2, N}$ and the odd modes $\Psi_{2, N+1}$ do not mix in the course of evolution. This suggests the existence of a double grading of the set of solutions. In particular one has the even and odd solutions:

$$
\Psi_{\text {even }}=\sum_{\mathscr{N}=-\infty}^{\infty} Z_{2, N} \Psi_{2 \mathscr{N}}, \quad \Psi_{\text {odd }}=\sum_{\mathscr{N}=-\infty}^{\infty} Z_{2 \mathscr{N}+1} \Psi_{2 \mathscr{N}+1}
$$

\section{MODE EXPANSION OF THE SOLUTIONS}

Consider expressing the solution $\Psi$ of the two-component Wheeler-DeWitt equation (5) as a linear combination of the eigenfunctions $\Psi_{\mathscr{N}}$ of the Hamiltonian: 


$$
\Psi=\sum_{\mathscr{N}=-\infty}^{\infty} Z_{\mathscr{N}} \Psi_{\mathscr{N}}, \quad Z_{\mathscr{N}}=Z_{\mathscr{N}}(\alpha)
$$

Substituting this equation in (5) and using Eqs. (9) and (10) to simplify the result, one finds

$$
\begin{gathered}
\sum_{n=0}^{\infty}\left[\left(\dot{\sigma}_{n}+i \sigma_{-n} E_{n}\right) \Phi_{n}+\sigma_{n} \dot{\Phi}_{n}\right]=0, \\
\sum_{n=0}^{\infty}\left[\left(\dot{\sigma}_{-n} E_{n}+i \sigma_{n} E_{n}^{2}+\sigma_{-n} \dot{E}_{n}\right) \Phi_{n}+\sigma_{-n} E_{n} \dot{\Phi}_{n}\right]=0
\end{gathered}
$$

where

$$
\sigma_{ \pm n}=\sigma_{ \pm n}(\alpha):=Z_{n} \pm Z_{-n}, \quad n=0,1,2, \ldots .
$$

Note that in general $Z_{-0} \neq Z_{0}$ and $\sigma_{-0} \neq \sigma_{0}$.

Next take the inner product of both sides of Eqs. (27) and (28) with $\Phi_{\tilde{n}}$. Making use of Eq. (23) and orthonormality of $\Phi_{n}$, one has:

$$
\begin{gathered}
\dot{\sigma}_{n}+i E_{n} \sigma_{-n}+\frac{3}{4} \sqrt{(n+1)(n+2)} \sigma_{n+2}-\frac{3}{4} \sqrt{n(n-1)} \sigma_{n-2}=0, \\
E_{n} \dot{\sigma}_{-n}+i E_{n}^{2} \sigma_{n}+\dot{E}_{n} \sigma_{-n}+\frac{3}{4} \sqrt{(n+1)(n+2)} E_{n+2} \sigma_{-(n+2)}-\frac{3}{4} \sqrt{n(n-1)} E_{n-2} \sigma_{-(n-2)}=0,
\end{gathered}
$$

where $\sigma_{-(0)}=\sigma_{-0}$.

In order to analyze these equations further, let us solve for $\sigma_{-n}$ from (29) and substitute the result in (30). Doing the same for $\sigma_{-(n \pm 2)}$, one obtains:

$$
\begin{gathered}
\sigma_{-n}=\frac{i}{E_{n}}\left[\dot{\sigma}_{n}+\frac{3}{4} \sqrt{(n+1)(n+2)} \sigma_{n+2}-\frac{3}{4} \sqrt{n(n-1)} \sigma_{n-2}\right], \\
\ddot{\sigma}_{n}+\left[E_{n}^{2}-\frac{3}{2}\left(n^{2}+n+1\right)\right] \sigma_{n}+\frac{3}{2} \sqrt{(n+1)(n+2)} \dot{\sigma}_{n+2}-\frac{3}{2} \sqrt{n(n-1)} \dot{\sigma}_{n-2} \\
+\left(\frac{3}{4}\right)^{2} \sqrt{(n+1)(n+2)(n+3)(n+4)} \sigma_{n+4}+\left(\frac{3}{4}\right)^{2} \sqrt{n(n-1)(n-2)(n-3)} \sigma_{n-4}=0 .
\end{gathered}
$$

Note that Eq. (32) links only $\sigma_{2 n}$ 's, with $n=0,1, \ldots$, thus making the analysis of the coefficients of non-negative modes independent of those of the negative modes.

Equations (31) and (32) clearly display the splitting of the solutions of the Wheeler-DeWitt equation into even and odd solutions. Since these equations are linear and there is no correlation between the even and odd solutions, every solution is a linear combination of an even and an odd solution. Hence without loss of generality, one can restrict to the analysis of $\Psi_{\text {even }}$ and $\Psi_{\text {odd }}$.

Before engaging in this analysis, however, I would like to note that the mode expansion (26) for the two-component wave function $\Psi$, translates into

$$
\psi=\sum_{n=0}^{\infty} \sigma_{n} \Phi_{n},
$$

for the one-component wave function. Thus, it is the coefficients of the zero and positive modes that determine the solution of the Wheeler-DeWitt equation (1). The negative modes are related to the derivative of $\psi$ with respect to $\alpha$. Hence, they are also uniquely determined once the zero and positive modes are given.

In the following sections, I shall show that the mode expansion of the solution of the Wheeler-DeWitt equation (1) is always infinite. This is another way of demonstrating the fact that the adiabatic approximation, which corresponds to including only a single mode in this expansion, always fails. Nevertheless, if one tries to blindly apply the adiabatic approximation or a slightly 
improved version of it in which one sets all $\sigma_{ \pm n}$ to zero, except $\sigma_{ \pm n_{*}}$ for some $n_{*}$, then Eqs. (29) and (30) yield $\ddot{\sigma}_{n *}+E_{n}^{2} \sigma_{n *}=0$. This corresponds to the eigenvalue equation for a onedimensional quantum particle of mass $1 / 2$ and potential $\mathscr{T}(\alpha):=-E_{n}^{2}=\kappa e^{4 \alpha}-(2 n+1) m e^{3 \alpha}$. Specifically, $\sigma_{n *}$ is the zero-energy eigenfunction. For $\kappa=1, \mathscr{V}$ vanishes at $\alpha=-\infty$. It decreases to attain a negative minimum as $\alpha$ increases, and then monotonically increases to $+\infty$ as $\alpha \rightarrow$ $+\infty$. Hence, if one requires the zero-energy eigenfunction to vanish at $\alpha=+\infty$ where $\mathscr{V}=+\infty$, then the mass $m$ must satisfy a peculiar quantization condition. This is analogous to the mass quantization advocated in Ref. 11. However, note that the basic hypothesis of this argument, namely the validity of the adiabatic approximation or alternatively the presence of only a single mode in the mode expansion (33), is never satisfied. Hence, the argument leading to the quantization of mass does not apply.

\section{EVEN SOLUTIONS}

Let us first prove:

Lemma 1: An even solution $\Psi_{\text {even }}=\Sigma_{\mathscr{N}} Z_{2} \Psi_{2 N}$ is nontrivial, i.e., $\Psi_{\mathrm{even}} \neq 0$, if and only if $\sigma_{ \pm 0} \neq 0$.

In order to prove this statement, one sets $n=0$ in Eqs. (29) and (30). This leads to

$$
\begin{gathered}
\dot{\sigma}_{0}+i E_{0} \sigma_{-0}+\frac{3 \sqrt{2}}{4} \sigma_{2}=0 \\
E_{0} \dot{\sigma}_{-0}+\dot{E}_{0} \sigma_{-0}+i E_{0}^{2} \sigma_{0}+\frac{3 \sqrt{2}}{4} E_{2} \sigma_{-2}=0
\end{gathered}
$$

Therefore, if $\sigma_{ \pm 0}=0$, both $\sigma_{2}$ and $\sigma_{-2}$ must vanish. However, by virtue of Eqs. (32) and (31), this is sufficient to conclude that $\sigma_{2 \mathscr{N}}=0$, for all $\mathscr{N} \in \mathbb{Z}$. This completes the proof of Lemma 1. Furthermore, one can prove

Lemma 2: Every nontrivial even solution has an infinite mode expansion.

The even solutions may nevertheless be constructed inductively in terms of $\sigma_{ \pm 0}$. This is done by noting that Eqs. (31) and (32) yield trivial algebraic equations for $\sigma_{ \pm 2(n+1)}$ in terms of $\sigma_{ \pm 2 n}$. Performing an induction on $n$, one can express the coefficients of all the modes as linear combinations of $\sigma_{ \pm 0}$ and its derivatives. For example, one has

$$
\begin{gathered}
\sigma_{2}=-\frac{1}{\sqrt{2}}\left(\frac{4}{3}\right)\left(\dot{\sigma}_{0}+i E_{0} \sigma_{-0}\right), \\
\sigma_{4}=\frac{1}{\sqrt{4 !}}\left(\frac{4}{3}\right)^{2}\left[\ddot{\sigma}_{0}+2 i E_{0} \dot{\sigma}_{-0}+\left(\frac{3}{2}-E_{0}^{2}\right) \sigma_{0}+2 i \dot{E}_{0} \sigma_{-0}\right] .
\end{gathered}
$$

It is easily seen that as a result of this construction $\sigma_{2 n}$ involves the first $n$ derivatives of $\sigma_{0}$, first $n-1$ derivatives of $\sigma_{-0}$, and the first $n-1-j$ derivatives of $E_{2 j}$, for $j=0,1, \ldots, n-1$.

Since according to Eq. (24), $\dot{E}_{n}$ diverges at the zero of $E_{n}$, demanding regularity of $\sigma_{n}$ would impose certain conditions on $\sigma_{ \pm 0}$. In fact, in view of the appearance of $E_{n}^{2}$ in Eq. (32), which is analytic for every $\alpha$, one can easily show that the only source of possible singularity is the term proportional to $E_{0}$ in the expression for $\sigma_{2}$. Thus, in order to ensure the regularity of $\sigma_{2 n}$, one must require that at the zero of $E_{0}$, i.e., $\alpha=\ln (\mathrm{m} / \kappa), \sigma_{-0} E_{0}$ and all its derivatives must vanish. In other words there must be an open neighborhood $\mathscr{O}$ of $\alpha=\ln (m / \kappa)$, in which $\sigma_{-0}(\alpha)$ is of the form $E_{0}(\alpha) f(\alpha)$ for some smooth $\left(C^{\infty}\right)$ function $f$. In particular $\sigma_{-0}(\ln (m / \kappa))$ must vanish. Note that for the flat $(\kappa=0)$ and open $(\kappa=-1)$ cases, this condition is void and $\sigma_{2 n}$ are regular if and only if $\sigma_{ \pm 0}$ are regular.

Finally note that termination of the mode expansion for a nontrivial even solution is equivalent to vanishing of at least three consecutive $\sigma_{2 n}$ 's. This would impose three functional conditions on $\sigma_{ \pm 0}$ which are in general incompatible. This proves Lemma 2. 


\section{ODD SOLUTIONS}

The structure of the odd solutions is similar to that of the even solutions. The role of $\sigma_{ \pm 0}$ is played by $\sigma_{ \pm 1}$. In particular, one has

Lemma 3: An odd solution $\Psi_{\text {odd }}=\sum_{\mathscr{N}} Z_{2 \mathscr{N}+1} \Psi_{2 \mathscr{N}+1}$ is nontrivial, if and only if $\sigma_{ \pm 1}:=Z_{1}$ $\pm Z_{-1} \neq 0$.

This is a direct consequence of Eqs. (29) and (32) which also provide an inductive construction of the mode coefficients $\sigma_{ \pm(2 n+1)}$ in terms of $\sigma_{ \pm 1}$. For example one has:

$$
\begin{gathered}
\sigma_{3}=-\frac{1}{\sqrt{3 !}}\left(\frac{4}{3}\right)\left(\dot{\sigma}_{1}+i E_{1} \sigma_{-1}\right) \\
\sigma_{5}=\frac{1}{\sqrt{5 !}}\left(\frac{4}{3}\right)^{2}\left[\ddot{\sigma}_{1}+2 i E_{1} \dot{\sigma}_{-1}+\left(\frac{9}{2}-E_{1}^{2}\right) \sigma_{1}+2 i \dot{E}_{1} \sigma_{-1}\right] .
\end{gathered}
$$

A natural consequence of this inductive construction is

Lemma 4: Every nontrivial odd solution has an infinite mode expansion.

The regularity condition on the odd solutions is analogous to the even case. One can again show that the only source of possible singularity is the term $E_{1} \sigma_{-1}$ in the expression for $\sigma_{3}$. Hence the regularity of $\sigma_{2 n+1}$ and therefore $\psi$ is ensured by requiring that in the vicinity of the zero of $E_{1}$, namely $\alpha=\ln (3 m / \kappa), \sigma_{-1}$ must be of the form $E_{1}(\alpha) g(\alpha)$, for some smooth function $g$.

The occurrence of two free functions of $\alpha$ in the construction of even and odd solutions is in complete agreement with the general structure of the Wheeler-DeWitt/Klein-Gordon equation (1) whose solutions depend on two boundary or two initial conditions. The latter are given by two functions of $\alpha$ which in the above procedure can be used to determine $\sigma_{ \pm 0}$ or $\sigma_{ \pm 1}$ depending on whether the solution is even or odd. In general the solution will be the sum of an even and an odd part. In this case the boundary (initial) conditions would determine both $\sigma_{ \pm 0}$ and $\sigma_{ \pm 1}$. Once these functions are determined the above iterative construction yields the mode expansion of the general solution.

\section{REAL SOLUTIONS}

Probably the best-known proposal for the solution of the Wheeler-DeWitt equation is that of Hartle and Hawking ${ }^{12}$ who suggested the path integral expression $\psi \propto \int \exp (-I)$ for the ground state wave function. Here $I$ stands for the Euclidean action. The Hartle-Hawking wave function is by construction real.

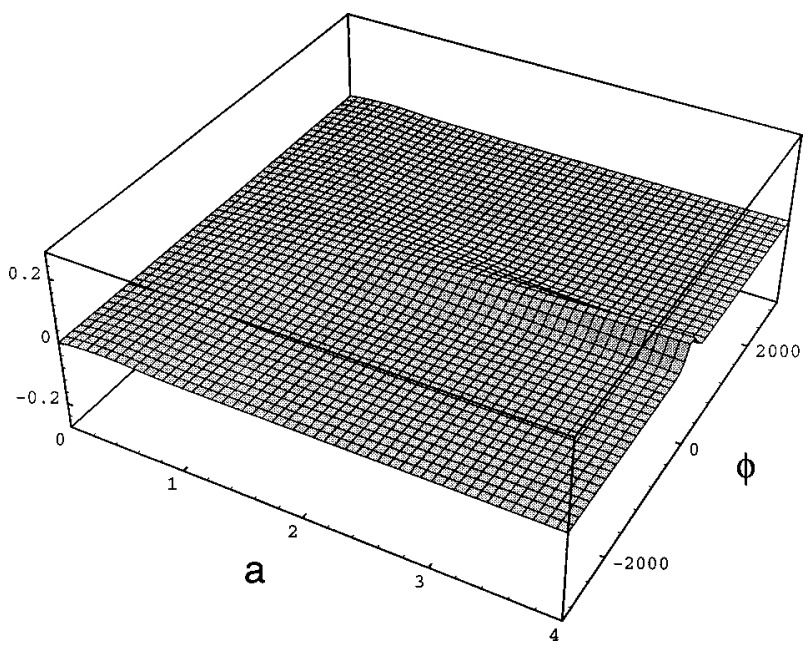

FIG. 1. Plot of $\psi_{0}=\psi_{2}=\Sigma_{n=0}^{2} \sigma_{n} \Phi_{n}$, for $\sigma_{ \pm 1}=\sigma_{-0}=0, \sigma_{0}=1$, and $m=10^{-6}$. 


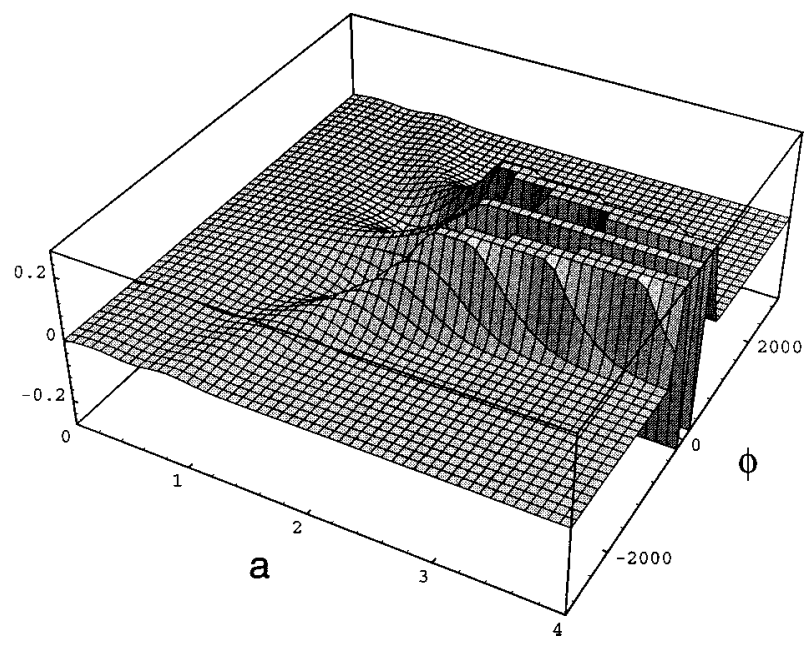

FIG. 2. Plot of $\psi_{4}:=\sum_{n=0}^{4} \sigma_{n} \Phi_{n}$, for $\sigma_{ \pm 1}=\sigma_{-0}=0, \sigma_{0}=1$, and $m=10^{-6}$.

It is not difficult to see that the reality of the one-component solution of the Wheeler-DeWitt equation implies the reality of the coefficient functions $\sigma_{n}$ in its mode expansion (33). This is because $\Phi_{n}$ are already real. In view of this observation and Eq. (29), $E_{n} \sigma_{-n}$ must be imaginary. However, as discussed in Sec. II for the open and flat universes $(\kappa=-1,0), E_{n}$ is always real so that $\sigma_{-n}$ must be imaginary. For the close universe $(\kappa=1) E_{n}$ is real for $a \leqslant m(2 n+1)$ and imaginary otherwise. Hence in this case $(\kappa=1)$ the opposite holds for $\sigma_{-n}$, i.e., it is imaginary for $a<m(2 n+1)$ and real for $a>m(2 n+1)$.

These conditions can also be stated as the reality condition on $\sigma_{0}, \sigma_{1}, \sigma_{2}$, and $\sigma_{3}$ which also generate the solutions. Therefore, they are rather trivial.

\section{APPROXIMATE SOLUTIONS}

As shown in the preceding sections, the mode expansion of the solutions never terminates. Therefore, one cannot use it to construct an exact solution unless one can actually find the generic expression for the mode coefficients and sum the series. This is an almost impossible task. However, one can terminate the mode expansion artificially and obtain an infinite class of approximate solutions. $\psi \approx \psi_{n_{*}}:=\sum_{n=0}^{n_{*}^{*}} \sigma_{n} \Phi_{n}$. The domain of reliability of this solution will in general depend on the boundary (initial) conditions, alternatively the functions $\sigma_{ \pm 0}$ and $\sigma_{ \pm 1}$.

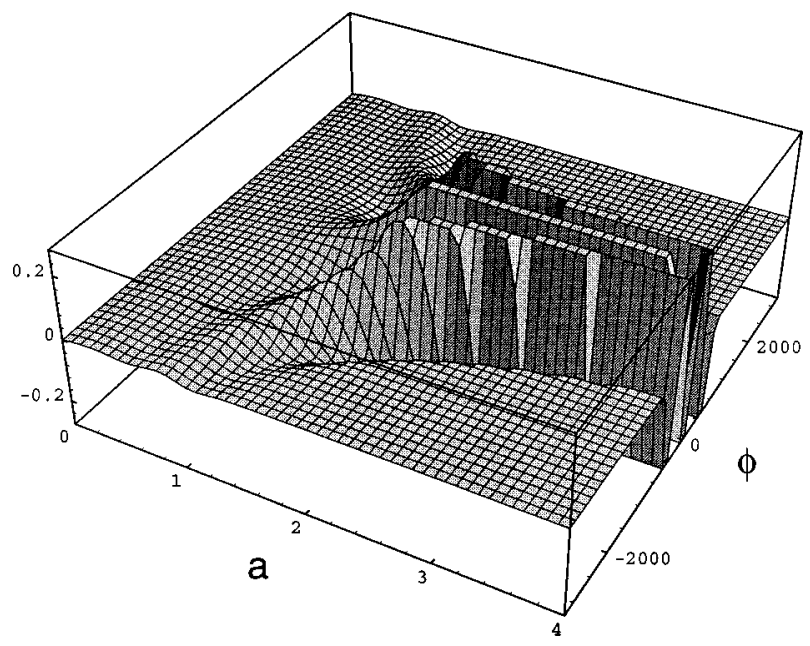

FIG. 3. Plot of $\psi_{6}:=\Sigma_{n=0}^{6} \sigma_{n} \Phi_{n}$, for $\sigma_{ \pm 1}=\sigma_{-0}=0, \sigma_{0}=1$, and $m=10^{-6}$. 


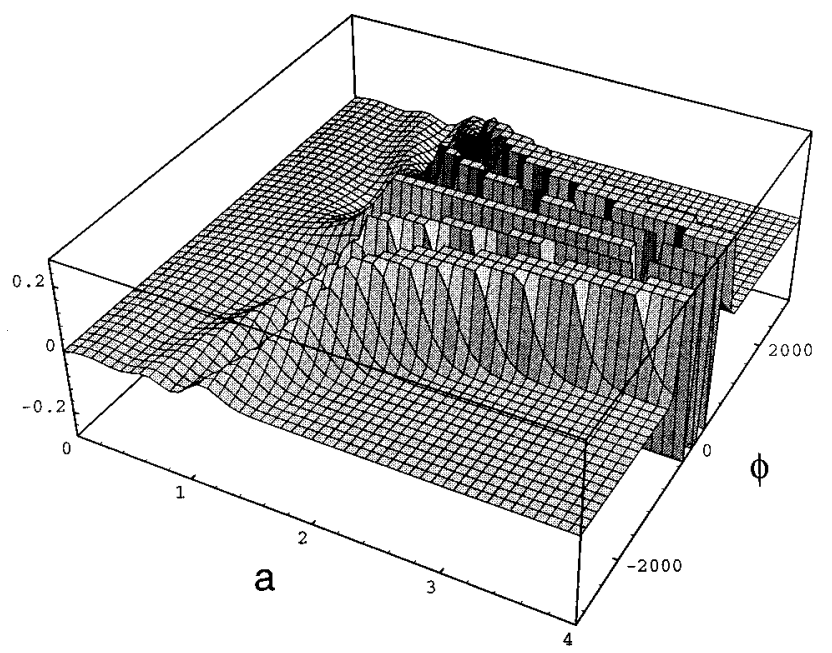

FIG. 4. Plot of $\psi_{8}:=\Sigma_{n=0}^{8} \sigma_{n} \Phi_{n}$, for $\sigma_{ \pm 1}=\sigma_{-0}=0, \sigma_{0}=1$, and $m=10^{-6}$.

In this section, I shall examine the simple choice $\sigma_{-0}=\sigma_{ \pm 1}=0$ and $\sigma_{0}=1$. Clearly, this corresponds to a real even solution whose mode coefficients can be obtained using Eq. (32). For example for the closed FRW case where $\kappa=1$, one has

$$
\begin{gathered}
\sigma_{2}=0, \quad \sigma_{4}=\left(\frac{2}{3}\right)^{5 / 2}\left(\frac{3}{2}+a^{4}-m a^{3}\right), \quad \sigma_{6}=\left(\frac{32 a^{3}}{81 \sqrt{5}}\right)(3 m-4 a), \\
\sigma_{8}=\frac{4}{243} \sqrt{\frac{2}{35}}\left(4 a^{8}-40 m a^{7}+36 m^{2} a^{6}+324 a^{4}-288 m a^{3}+189\right), \\
\sigma_{10}=-\frac{16}{10935 \sqrt{7}}\left(96 a^{5}-1000 m a^{4}+744 m^{2} a^{3}+3604 a-2655 m\right) a^{3} .
\end{gathered}
$$

Substituting these relations in the expression for $\psi_{n}$ and making use of the identity ${ }^{13}$

$$
H_{n}(x)=2^{n} x^{n}+\sum_{i=1}^{[n / 2]}(-1)^{i} 2^{n-i}(2 i-1) ! !\left(\begin{array}{c}
n \\
2 i
\end{array}\right) x^{n-2 i}
$$

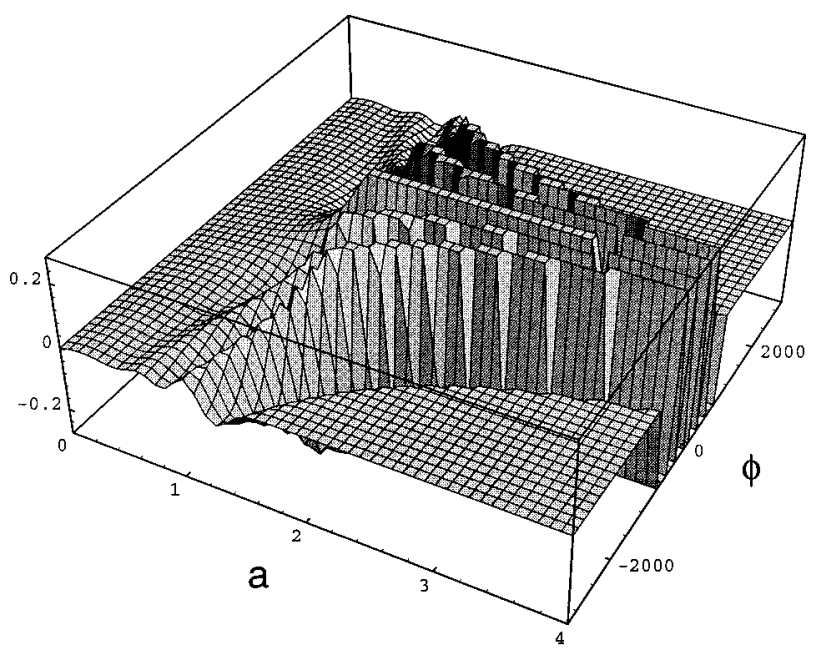

FIG. 5. Plot of $\psi_{10}:=\sum_{n=0}^{10} \sigma_{n} \Phi_{n}$, for $\sigma_{ \pm 1}=\sigma_{-0}=0, \sigma_{0}=1$, and $m=10^{-6}$. 


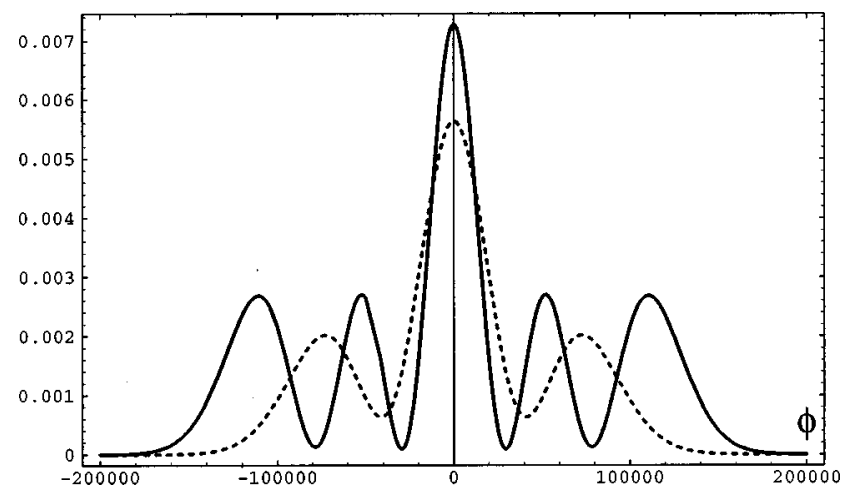

FIG. 6. Plot of the restriction of $\psi_{6}$ (dotted line), $\psi_{8}$ (dashed line), and $\psi_{10}$ (full line) to the $a=0.1$ line. Note that the graphs of $\psi_{8}$ and $\psi_{10}$ coincide for this value of $a$. This shows the reliability of the finite mode approximation. Note also the range of the values of $\phi$ over which $\psi_{n}$ are nonzero.

to calculate the mode functions $\Phi_{n}$, one can compute $\psi_{0}=\psi_{2}, \psi_{4}, \psi_{6}, \psi_{8}$ and $\psi_{10}$. Figures 1-5 illustrate three-dimensional plots of these functions. Figures 6-9 are plots of the restriction of $\psi_{6}$, $\psi_{8}$, and $\psi_{10}$ to the lines $a=0.1,1,10,100$. These figures show that for small $a$ the finite mode approximation is quite reliable. In fact, as depicted in Fig. 6, for $a=0.1$, the graphs of $\psi_{8}$ and $\psi_{10}$ coincide. For larger $a$ one may improve the approximation by including the contribution of higher modes.

Note also that initially $\psi_{n}$ are nonzero for a wide range of values of $\phi$. However, as $a$ increases the width of this region, which is symmetric with respect to the line $\phi=0$, shrinks. This is easily seen from Figs. 6-9. Note also that for large $a$, the solutions picks about $\phi=0$ and the approximate solutions become less reliable. As $a \rightarrow \infty$, all the approximate solutions seem to vanish except at $\phi=0$.

\section{DISCUSSION AND CONCLUSION}

Probably the most important aspect of the two-component formulation of the WheelerDeWitt equation is the fact that the construction of the solutions in this formulation does not, $a$ priori, require fixing a particular inner product or boundary conditions. The freedom in the choice of the inner product is reflected as the independence of the mode expansion from the choice of the Hermitian matrix $h$ which together with the $L^{2}$ inner product on $L^{2}(\mathbb{R})$ determines the inner product on $\mathscr{H}=L^{2}(\mathbb{R}) \oplus L^{2}(\mathbb{R})$. As far as the boundary conditions are concerned, besides the implicit restriction of vanishing boundary condition at "spatial" infinity, the analysis presented here applies generally.

An interesting consequence of the application of the two-component formalism is a specific double grading of the solutions of the Wheeler-DeWitt equation. This allows one to write down

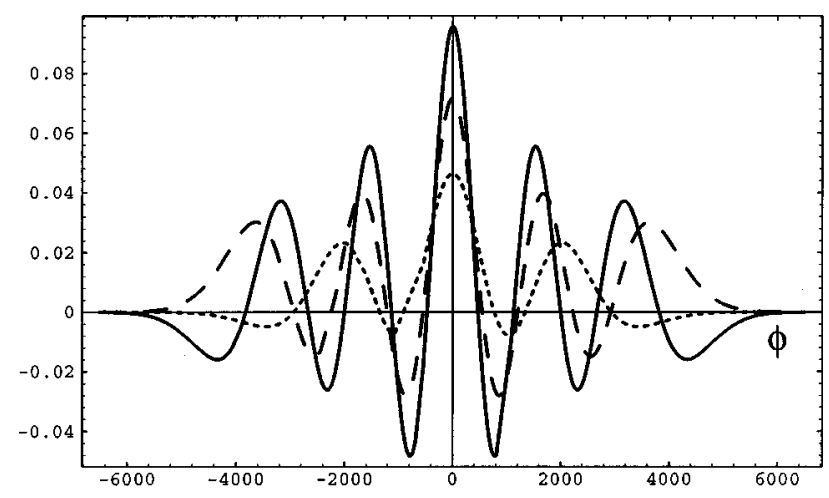

FIG. 7. Plot of the restriction of $\psi_{6}$ (dotted line), $\psi_{8}$ (dashed line), and $\psi_{10}$ (full line) to the $a=1$ line. Note the range of the values of $\phi$ over which $\psi_{n}$ are nonzero. 


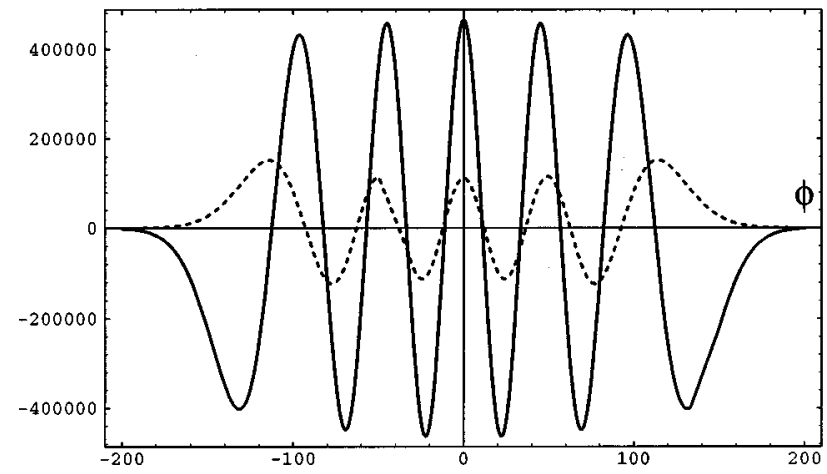

FIG. 8. Plot of the restriction of $\psi_{8}$ (dotted line), and $\psi_{10}$ (full line) to the $a=10$ line. Note the range of the values of $\phi$ over which $\psi_{n}$ are nonzero.

the most general solution of this equation as a sum of definite-parity (even and odd) solutions whose structure can be easily understood. The two definite-parity parts of the solution do not mix during the evolution. In particular, if the initial wave function has definite parity, its parity remains intact throughout the evolution.

Unfortunately, the mode expansion of the solutions never terminates. Thus, a closed expression for an exact solution cannot be found in this way. However, approximate solutions can be constructed by artificially terminating the mode expansion. The domain of applicability of such approximations depend generally on the boundary (initial) conditions.

An alternative approximation is the adiabatic approximation which is, however, not valid. The ansatz of the adiabatic approximation, i.e., $\psi \approx \sigma_{n}(\alpha) \Phi_{n}(\alpha, \phi)$, is reminiscent of the application of the Born-Oppenheimer approximation in quantum cosmology originally advocated by Banks. ${ }^{14}$ In this approach one views the gravitational degrees of freedom as the "slow" variables and the matter degrees of freedom as the "fast" variables and considers solutions of the form $\psi=\psi_{\text {gravity }}(\alpha) \psi_{\text {matter }}(\alpha, \phi)$. The results of this article indicate that for $\psi_{\text {matter }}=\Phi_{n}$ such an approximation cannot be valid.

Another notable implication of the results of this article is regarding the implicit boundary condition at the initial singularity $a=0$. If one assumes that the coefficient functions $\sigma_{n}$ are regular at $a=0$, then as a consequence of the appearance of $a^{3 / 4}$ in the expression for $\Phi_{n}$, the onecomponent wave-function $\psi$ vanishes at $a=0$. This is much in the spirit of an argument of DeWitt. ${ }^{15}$ Here it follows as a direct consequence of a regularity condition (which may or may not hold). As seen from Figs. 1 to 5, the choice $\sigma_{-0}=\sigma_{ \pm 1}=0$ and $\sigma_{0}=1$ leads to approximate solutions which are regular at $a=0$. They clearly satisfy DeWitt's boundary conditions.

A problem which is not addressed in this article is that of the convergence of the mode expansion in $\mathscr{H}$. This is a difficult problem and I have not been able to find the necessary and sufficient conditions under which the mode expansion converges. In fact, a rigorous convergence

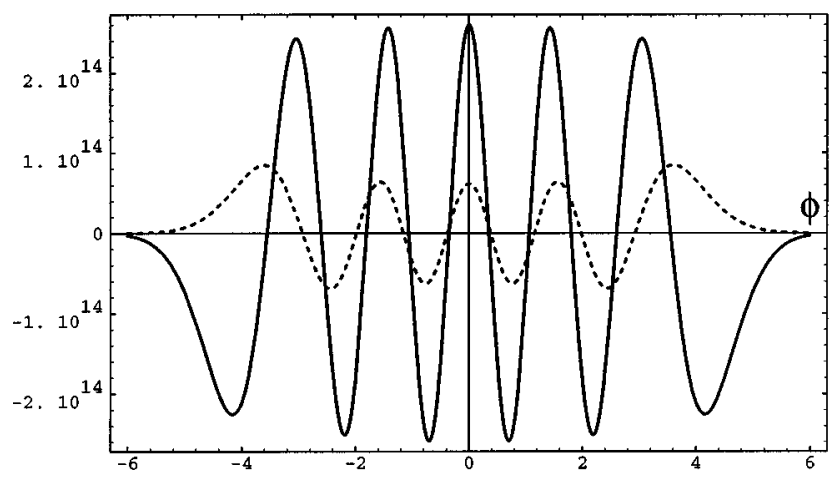

FIG. 9. Plot of the restriction of $\psi_{8}$ (dotted line), and $\psi_{10}$ (full line) to the $a=100$ line. Note the range of the values of $\phi$ over which $\psi_{n}$ are nonzero. 
analysis requires a choice of a positive-definite inner product (norm) on $\mathscr{H}$. However, as demonstrated in Sec. IX, there are regions of the minisuperspace in which the contribution from the higher order modes is small. This may be viewed as an indication of possible pointwise asymptotic convergence of the mode expansion in this regions. This argument is however far from being satisfactory and further investigation of this point is necessary.

Finally, I wish to emphasize the following points.

(1) The fact that the two-component Hamiltonian (6) belongs to the dynamical Lie algebra $\mathrm{su}_{s}(1,1) \otimes \mathrm{su}_{u}(1,1)$ suggests a further investigation of the solution of the corresponding Schrödinger equation using the group theoretical methods such as those employed in solving the Schrödinger equation for time-dependent harmonic oscillators. ${ }^{16}$ Recall that the Hamiltonian for a time-dependent harmonic oscillator belongs to $\mathrm{su}_{u}(1,1){ }^{6}$

(2) The two-component approach to Wheeler-DeWitt equation has a wider domain of applicability than the simple minisuperspace model studied in this article. For example, the addition of an interaction potential for the scalar field would only complicate the defining equation for $\Phi_{n}$ and the inductive construction of the mode expansion. The general structure of the method would however remain intact. In particular, the $\phi$-dependence of the solutions would still be given by $\Phi_{n}$. More generally, for any other minisuperspace, in the gauge in which the shift vector corresponding to DeWitt's supermetric ${ }^{15}$ vanishes, the two-component Hamiltonian is of the form (6), where $D$ is some second-order elliptic operator. Therefore, even in this case the developments reported in this article are essentially applicable.

Note added in proof: After the completion of this work, Refs. 17, 18 were brought to my attention where the authors use two different two-component formulations of the Wheeler-DeWitt equation. The methods described in these articles differ substantially from the present article's. I have also noticed a recent paper by Embacher ${ }^{19}$ who defines the operator $D$ of Eq. (7) as the (matter) energy operator and obtains the mode expansion (33) in the context of the one-component Wheeler-DeWitt equation. He does not, however, obtain the equations satisfied by the mode coefficients. Instead, he focuses on the semiclassical and adiabatic approximations. The twocomponent approach developed in this paper justifies the choice of the mode functions made by Embacher in a very natural way. It also makes it possible to obtain the structure of the mode expansion by iteratively solving for the mode coefficients.

\section{ACKNOWLEDGMENTS}

I would like to thank B. Darian, M. Razavi, and especially D. Page for invaluable comments and suggestions. I would also like to acknowledge the financial support of the Killam Foundation of Canada.

${ }^{1}$ D. N. Page, in Gravitation: A Banff Summer Institute, edited by R. Mann and P. Wesson (World Scientific, Singapore, 1991); D. L. Wiltshire, in Cosmology: The Physics of the Universe (World Scientific, Singapore, to be published).

${ }^{2}$ D. J. Kaup and A. P. Vitello, Phys. Rev. D 9, 1648 (1974).

${ }^{3}$ S. W. Hawking and D. N. Page, Phys. Rev. D 42, 2655 (1990); see also A. Mostafazadeh, Nucl. Phys. B 509, 529 (1998), and references therein.

${ }^{4}$ A. Mostafazadeh (unpublished).

${ }^{5}$ H. Feshbach and F. Villars, Rev. Mod. Phys. 30, 24 (1958); B. R. Holstein, Topics in Advanced Quantum Mechanics (Addison-Wesley, Redwood City, CA, 1992).

${ }^{6}$ R. Jackiw, Int. J. Mod. Phys. A 3, 285 (1988).

${ }^{7}$ L. I. Schiff, Quantum Mechanics (McGraw-Hill, New York, 1955).

${ }^{8}$ A. Mostafazadeh, Phys. Rev. A 55, 1653 (1997).

${ }^{9}$ A. Mostafazadeh, Phys. Lett. A 228, 7 (1997).

${ }^{10}$ M. Read and B. Simon, Functional Analysis (Academic, San Diego, 1980), Vol. I.

${ }^{11}$ C. Kiefer, Phys. Rev. D 38, 1761 (1988).

${ }^{12}$ J. B. Hartle and S. W. Hawking, Phys. Rev. D 28, 2960 (1983).

${ }^{13}$ I. S. Gradshteyn and I. M. Ryzhik, Table of Integrals, Series, and Products (Academic, New York, 1980).

${ }^{14}$ T. Banks, Nucl. Phys. B 249, 332 (1985); R. Brout, Found. Phys. 17, 603 (1987); R. Brout and G. Venturi, Phys. Rev. D 39, 2436 (1989); G. Venturi, in Differential Geometric Methods in Theoretical Physics, edited by L.-L. Chau and W. Nahm (Plenum, New York, 1990); D. P. Datta, Phys. Rev. D 48, 5746 (1993).

${ }^{15}$ B. S. DeWitt, Phys. Rev. 160, 1113 (1967).

${ }^{16}$ H. R. Lewis, Jr. and W. B. Riesenfeld, J. Math. Phys. 10, 1458 (1969); D. M. Greenberger, ibid. 15, 395 (1974); R. W. Hasse, ibid. 16, 2005 (1975); V. V. Dodonov and V. I. Man'ko, Phys. Rev. A 20, 550 (1979); L. F. Landovitz, A. M. Levine, and W. M. Schreiber, ibid. 20, 1162 (1979); M. S. Abdalla and R. K. Colegrave, ibid. 32, 1958 (1985); A. B. Nassar and R. T. Berg, ibid. 34, 2462 (1986); C. M. Cheng and P. C. W. Fung, J. Phys. A 21, 4115 (1988); F. Wolfs and H. J. Korsch, Phys. Rev. A 37, 1934 (1988); G. S. Agarwal and S. Arun Kumar, Phys. Rev. Lett. 67, 3665 (1991); A. de 
Souza Dutra, C. F. de Souza, and L. C. de Albuquerque, Phys. Lett. A 156, 371 (1991); S. P. Kim, J. Phys. A 27, 3927 (1994); J.-Y. Ji and J. K. Kim, Phys. Rev. A 51, 4268 (1995); A. Mostafazadeh, ibid. 55, 4084 (1997).

${ }^{17}$ S. P. Kim, Phys. Rev. D 46, 3403 (1992).

${ }^{18}$ T. Dereli, M. Önder, and R. W. Tucker, Phys. Lett. B 324, 134 (1994).

${ }^{19}$ Franz Embacher, Nucl. Phys. B 479, 461 (1996). 